\title{
Phenotypic and molecular effects of chronic gamma irradiation on Curcuma alismatifolia
}

\begin{abstract}
Mutation breeding is one of the methods for generating genetic variation and obtaining new cultivars of ornamental plants during the past decades. In present study, the effects of four doses $(0,14.6,33$, and $87.4 \mathrm{~Gy})$ of chronic gamma irradiation on three cultivars of Curcuma alismatifolia and one Curcuma hybrid were investigated. Morphological aberrations from non-treated plants were observed by exposing growing plants. Higher doses induced phenotypical variations and significantly affected the plant growth parameters and flowering capacity. In terms of genetic variation, among the irradiated cultivars, the number of presumed alleles revealed by SSR analysis ranged from two to five with a mean value of 3.1 to 3.7 alleles per locus for radiation doses. The average value of the effective number of alleles, Nei's gene diversity, and Shannon's information index were 2.42-2.66, 0.50-0.56, and $0.90-1.03$, respectively. Heat map hierarchical clustering divided 52 studied individuals into four major clusters. Results of this study showed that chronic gamma irradiation efficiently can enhance the phenotypical and genetic variations in $C$. alismatifolia cultivars at doses of 33 Gy and 84.6 Gy. In addition, SSR markers will likely accelerate the progress of selection of desired mutants during mutation breeding programs.
\end{abstract}

Keyword: Genetic variation; Mutation breeding; Microsatellite markers; Morphological variation; Zingiberaceae 